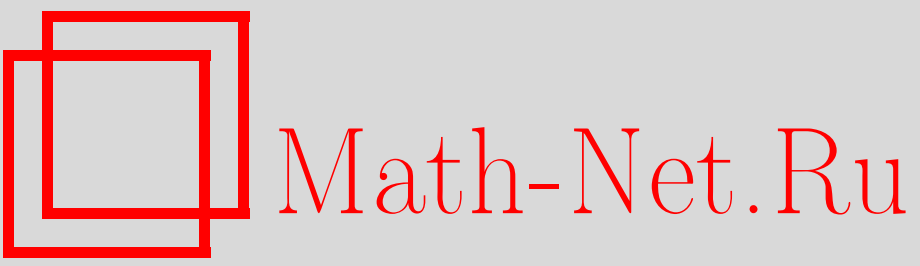

М. Н. Крейн, Обобщение теоремы об эквивалентности координатного и алгебраического определений гладкого многообразия, Итоги науки и техн. Сер. Соврем. мат. и ее прил. Темат. обз., 2019, том 171, 114-117

DOI: https://doi.org/10.36535/0233-6723-2019-171-114-117

Использование Общероссийского математического портала Math-Net.Ru подразумевает, что вы прочитали и согласны с пользовательским соглашением

http: //www. mathnet.ru/rus/agreement

Параметры загрузки:

IP : 18.234 .197 .8

26 апреля 2023 г., 13:23:54 


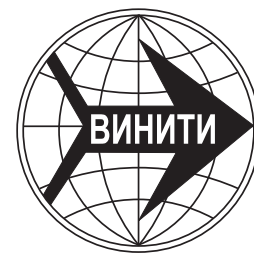

ИТОГИ НАУКИ И ТЕХНИКИ.

Современная математика и ее приложения.

Тематические обзоры.

Том 171 (2019). С. 114-117

DOI: 10.36535/0233-6723-2019-171-114-117

УДК 512.552, 515.126

\title{
ОБОБЩЕНИЕ ТЕОРЕМЫ ОБ ЭКВИВАЛЕНТНОСТИ КООРДИНАТНОГО И АЛГЕБРАИЧЕСКОГО ОПРЕДЕЛЕНИЙ ГЛАДКОГО МНОГООБРАЗИЯ
}

\author{
(c) 2019 г. $\quad$ M. Н. КРЕЙН
}

\begin{abstract}
АннотАция. В работе обобщена теорема об эквивалентности координатного и алгебраического определения гладкого многообразия. При алгебраическом определении точкой называют гомоморфизм из алгебры гладких действительных функций, заданных на многообразии, в поле действительных чисел. Рассматривается обобщение для случая, когда поле действительных чисел заменяется на произвольную ассоциативную нормированную алгебру, в общем случае некоммутативную.
\end{abstract}

Ключевъе слова: ассоциативная алгебра, многообразие, гомоморфизм алгебр, центральная алгебра.

\section{GENERALIZATION OF A THEOREM ON THE EQUIVALENCE OF THE COORDINATE AND ALGEBRAIC DEFINITIONS OF A SMOOTH MANIFOLD}

\author{
(C) 2019 M. N. KREIN
}

\begin{abstract}
In this paper, we generalize the theorem on the equivalence of the coordinate and algebraic definitions of a smooth manifold. Within the framework of the algebraic approach, a point is considered as a homomorphism from the algebra of smooth real functions defined on a manifold into the field of real numbers. We consider a generalization for the case where the field of real numbers is replaced by an arbitrary associative normalized algebra, generally speaking, noncommutative.
\end{abstract}

Keywords and phrases: associative algebra, manifold, homomorphism of algebras, central algebra. AMS Subject Classification: 47A99

Пусть $K$ - нормированная алгебра, в общем случае некоммутативная, над полем $k$, содержащем поле действительных чисел $\mathbb{R}$, с открытым множеством обратимых элементов (например, операторная алгебра), $M$ - действительное конечномерное многообразие, $\mathcal{F}=C_{K}(M)$ - алгебра непрерывных функций из $M$ в $K$ с поточечно определенными операциями, которая также некоммутативна, если некоммутативна $K,|\mathcal{F}|$-множество $k$-гомоморфизмов из $\mathcal{F}$ в $K$ с базой топологии $\left\{f^{-1}(V) \mid V \subset K\right.$ открыто, $\left.f \in|\mathcal{F}|\right\}, \theta: M \rightarrow|\mathcal{F}|$ - отображение, задаваемое формулой $[\theta(p)](f)=f(p)$.

Теорема 1. Справедливы следующие утверждения.

1. Отображение $\theta$ инвективно.

2. Всякий гомоморфизм из $|\mathcal{F}|$, переводящий действительные функиии в действительные элементы из $K$, представляется в виде суммы $\theta(p)$ для некоторого $p \in M$ и отображения $\beta$, все значения которого необратимы в $K$. 


\section{3. Точка $p \in M$ определена однозначно.}

Под действительными функциями здесь понимаются функции, принимающие значения в поле $\mathbb{R}$, вложенном в $K$.

Если в этих обозначениях вместо $K$ и $k$ берется поле действительных чисел, $\theta$ оказывается взаимно однозначным отображением, даже гомеоморфизмом. Это является одним из основополагающих фактов diffiety-теории (см. [?, теорема 7.2]).

Доказательство теоремы 1.

1. Инъективность отображения $\theta$ следует из того факта, что, поскольку $M$ хаусдорфово, для различных точек $p, q \in M$ найдется функция $f \in \mathcal{F}$ с различными значениями $f(p)$ и $f(q)$, откуда $[\theta(p)](f) \neq[\theta(q)](f)$.

2. Выберем в $\mathcal{F}$ такую действительную функцию $\varphi$, что прообраз каждого ее значения компактен в $M$. (Условия, наложенные на алгебру $K$, обеспечивают включение множества действительных гладких функций в $\mathcal{F}$.) В [?, предложение п. 5.17] доказано, что такая действительная функция существует. Пусть $\alpha \in|\mathcal{F}|, \alpha: \mathcal{F} \rightarrow K$ - некоторый гомоморфизм, $\alpha(\varphi)=\lambda \in \varphi(M) \subset \mathbb{R}$ и $L=\varphi^{-1}(\lambda)$. Предположим, что для любого $x \in L$ найдется такое $f_{x} \in \mathcal{F}$, что $\left[\alpha\left(f_{x}\right)-f_{x}(x)\right]$ обратимо. Тогда найдется такой элемент $c_{x} \in K$, что $c_{x}\left[\alpha\left(f_{x}\right)-f_{x}(x)\right]=1$. Так как множество обратимых элементов открыто, то для некоторого $\varepsilon>0$ в $K$ существует $\varepsilon$-окрестность единицы, состоящая из обратимых элементов. (Тогда $\varepsilon n$-окрестность для для $n \in \mathbb{R}$ состоит из обратимых элементов.) В силу непрерывности функции $f_{x}$, а значит, и функции $c_{x}\left[\alpha\left(f_{x}\right)-f_{x}\right]$, в $M$ найдется открытая окрестность $U_{x}$ точки $x$, в которой значения последней функции лежат в указанной $\varepsilon$ окрестности единицы. Окрестности $\left\{U_{x}\right\}_{x \in L}$ образуют открытое покрытие множества $L$, и, в силу компактности $L$, из него можно выбрать конечное подпокрытие $U_{x_{1}}, \ldots, U_{x_{n}}$. Определим функцию

$$
g(x)=(\varphi(x)-\lambda)^{2}+\sum_{i=1}^{n}\left[c_{x_{i}}\left(\alpha\left(f_{x_{i}}\right)-f_{x_{i}}(x)\right)\right]
$$

Первое слагаемое здесь действительно, т.е. лежит в $\mathbb{R} \subset K$, и неотрицательно. Для любой точки $x \in M$ имеем

$$
\begin{aligned}
\|g(x)\|=\left\|\left[(\varphi(x)-\lambda)^{2}+n\right]+\left\{\sum_{i=1}^{n}\left[c_{x_{i}}\left(\alpha\left(f_{x_{i}}\right)-f_{x_{i}}(x)\right)\right]-n\right\}\right\| & \leqslant \\
& \leqslant\left[(\varphi(x)-\lambda)^{2}+n\right]+\sum_{i=1}^{n} \|\left[c_{x_{i}}\left(\alpha\left(f_{x_{i}}\right)-f_{x_{i}}(x)\right)-1 \| .\right.
\end{aligned}
$$

Поскольку

$$
\sum_{i=1}^{n}\left\|c_{x_{i}}\left(\alpha\left(f_{x_{i}}\right)-f_{x_{i}}(x)\right)-1\right\| \leqslant \varepsilon n \leqslant \varepsilon n_{x},
$$

где $n_{x}=(\varphi(x)-\lambda)^{2}+n$, заключаем, что $g(x)$ лежит в $\left(\varepsilon n_{x}\right)$-окрестности действительной положительной точки $n_{x}$, поэтому $g(x)$ обратимо при любом $x \in M$. Следовательно, на $M$ определена функция $\frac{1}{g}$ :

$$
\left(\frac{1}{g}\right)(x)=[g(x)]^{-1}
$$

Для любой точки $x \in M$ имеем

$$
\left(g \cdot \frac{1}{g}\right)(x)=g(x) \cdot[g(x)]^{-1}=1,
$$

т.е. $g \cdot \frac{1}{g}$ - единица алгебры $\mathcal{F}$, и

$$
\alpha(g) \cdot \alpha\left(\frac{1}{g}\right)=\alpha\left(g \cdot \frac{1}{g}\right)=\alpha(1)=1 .
$$


Значит, $\alpha(g)$ - обратимый элемент, потому не равен нулю. В то же время

$$
\alpha(g)=\alpha\left[(\varphi-\lambda)^{2}+\sum_{i=1}^{n}\left[c_{x_{i}}\left(\alpha\left(f_{x_{i}}\right)-f_{x_{i}}\right)\right]\right]=[\alpha(\varphi)-\lambda]^{2}+\sum_{i=1}^{n}\left\{c_{x_{i}}\left[\alpha\left(f_{x_{i}}\right)-\alpha\left(f x_{i}\right)\right]\right\}=0
$$

противоречие. Следовательно, предположение неверно, и существует такая точка $x_{\alpha} \in L$, что для всех $f \in \mathcal{F}$ отображение $\alpha(f)-f\left(x_{\alpha}\right)=\alpha(f)-\left[\theta\left(x_{\alpha}\right)\right](f)$ необратимо; здесь $\beta=\alpha-\theta\left(x_{\alpha}\right)$.

3. Пусть отображение $\alpha \in|\mathcal{F}|$ переводит действительные функции в действительные элементы алгебры $\mathcal{F}, \alpha=\theta(p)+\beta$, т.е. $\beta=\alpha-\theta(p)$. Так как все значения $\beta$ необратимы, а среди действительных элементов необратим только нуль, то для всякой действительной функции $f \in \mathcal{F}$ имеем $\beta(f)=0$, поэтому $\alpha(f)=\theta(p)(f)=f(p)$. Далее повторяем рассуждения доказательства п. 1 , а именно: для различных точек $p, q \in M$ найдется действительная функция $f \in \mathcal{F}$ с различными значениями $f(p)$ и $f(q)$, откуда вытекает, что $[\theta(p)](f) \neq[\theta(q)](f)$.

Теорема доказана.

В п. 2 формулировки теоремы 1 на рассматриваемые гомоморфизмы накладывается условие, используемое в доказательстве и состоящее в том, что они переводят действительные функции в действительные элементы из $K$. Действительные функции, очевидно, принадлежат центру алгебры $\mathcal{F}$, поэтому их образ при любом гомоморфизме должен лежать также в центре алгебры $K$.

Алгебра линейных операторов конечномерного пространства над $k$ является центральной. Это означает, что ее центр совпадает с полем $k$, т.е. с множеством операторов вида $\lambda I$, где $\lambda \in k$, а $I-$ тождественный оператор. Действительно, элементы центра по определению перестановочны со всеми элементами алгебры, значит, и с автоморфизмами, поэтому $A X=X A$ или $X=A^{-1} X A$ для всякого $X$, принадлежащего центру, и всякого автоморфизма $A$. Следовательно, матрица оператора не меняется при любом изменении базиса, что верно только для операторов вида $\lambda I$.

Оказывается, аналогичное свойство верно и в гильбертовом пространстве.

Теорема 2. Алгебра линейных ограниченных операторов гилъбертова пространства является иентральной.

Доказательство. Пусть $K=B(H)$ - алгебра линейных ограниченных операторов гильбертова пространства $H, X$ - оператор, принадлежащий ее центру.

1. Покажем, что любой вектор из $H$ является собственным для $X$. Пусть $e_{1} \in H$. Предположим, что $e_{2}=X\left(e_{1}\right) \neq \lambda e_{1}$ для любого $\lambda \in k$; тогда $e_{1}$ и $e_{2}$ линейно независимы. Построим такой автоморфизм $A$, что $X\left(e_{1}\right) \neq\left(A^{-1} X A\right)\left(e_{1}\right)$. Положим $A\left(e_{1}\right)=e_{1}$, выберем $e_{3} \neq \mu e_{2}$ и $e_{3} \neq \lambda e_{1}$ ни для каких $\lambda$ и $\mu$ из $k$ и положим $A\left(e_{3}\right)=e_{2}, A\left(e_{2}\right)=e_{3}$. Векторы $e_{1}, e_{2}, e_{3}$ линейно независимы, $L=L\left(e_{1}, e_{2}, e_{3}\right)$ - конечномерное подпространство, имеющее в гильбертовом пространстве $H$ имеет прямое дополнение $H_{1}$ (например, ортогональное дополнение). Пусть $A_{1}$ - какой-либо автоморфизм на $H_{1}$. Тогда $A=\left.A\right|_{L} \oplus A_{1}$. Отображение $\left.A\right|_{L}$ задано выше на базисных элементах $L$, $A$ - автоморфизм на $H, L$ и $A_{1}$-инвариантные подпространства для $A$. При этом

$$
\left(A^{-1} X A\right)\left(e_{1}\right)=A^{-1}\left(X\left(A\left(e_{1}\right)\right)=A^{-1}\left(X\left(e_{1}\right)\right)=A^{-1}\left(e_{2}\right)=e_{3} \neq e_{2},\right.
$$

следовательно, $A^{-1} X A \neq X$. Значит, предположение неверно, и для любого $e_{1} \in H$ найдется такое $\lambda \in k$, что $X\left(e_{1}\right)=\lambda e_{1}$.

2. Покажем, что это собственное значение единственно. Предположим, что $X\left(e_{1}\right)=\lambda_{1} e_{1}$, $X\left(e_{2}\right)=\lambda_{2} e_{2}, \lambda_{1} \neq \lambda_{2}$. Тогда $e_{1}$ и $e_{2}$ линейно независимы, и в качестве автоморфизма $A$ можно взять автоморфизм, переставляющий местами $e_{1}$ и $e_{2}$ и тождественный на дополнении к $L\left(e_{1}, e_{2}\right)$. Очевидно, $A^{-1}=A$. Получим

$$
\left(A^{-1} X A\right)\left(e_{1}\right)=A^{-1}\left(X\left(A\left(e_{1}\right)\right)\right)=A^{-1}\left(X\left(e_{2}\right)\right)=A^{-1}\left(\lambda_{2} e_{2}\right)=\lambda_{2} A^{-1}\left(e_{2}\right)=\lambda_{2} e_{1} \neq \lambda_{1} e_{1}=X\left(e_{1}\right),
$$

т.е. $A^{-1} X A \neq X$. Следовательно предположение неверно, и $\lambda_{1}=\lambda_{2}$. Теорема доказана.

Теорема 3 (следствие теорем 1 и 2). Пусть $K$-алгебра линейных операторов конечномерного пространства над $k$ или алгебра линейных ограниченных операторов гильбертова пространства, $М$ - конечномерное многообразие. Тогда справедливы следующие утверждения.

1. Отображение $\theta$ ингективно. 
2. Всякий гомоморфизм из $|\mathcal{F}|$ представляется в виде суммы $\theta(p)$ для некоторого $p \in M u$ гомоморфизма $\beta$, все значения которого необратимы в $K$.

3. Точка $p \in M$ определена однозначно.

\section{СПИСОК ЛИТЕРАТУРЫ}

1. Неструев Дж. Гладкие многообразия и наблюдаемые. - М.: МЦНМО, 2000.

Крейн Мария Нехемьевна

Липецкий государственный педагогический университет

E-mail: kreyn_mara@mail.ru 